\title{
Maintaining Nursing Students' Moral Sensitivity Through the Ethical Decision-making and Teacher's Support in Taiwan
}

Hsiao Lu Lee, RN, Midwife, Ph D.

Assistant Professor, Department of Nursing, Tajen University, No.20, Weixin Rd., Yanpu Township, Pingtung County 90741, Taiwan, R.O.C.

Article Details
Article Type: Research Article
Received date: $23^{\text {th }}$ January, 2021
Accepted date: $15^{\text {th }}$ March, 2021
Published date: $22^{\text {nd }}$ March, 2021

*Corresponding Author: Hsiao Lu Lee, RN, Midwife, Ph D, Assistant Professor, Department of Nursing, Tajen University, No.20, Weixin Rd., Yanpu Township, Pingtung County 90741, Taiwan, R.O.C. E-mail: 1ulu@tajen.edu.tw

Citation: Lee, H.L. (2021). Maintaining Nursing Students' Moral Sensitivity Through the Ethical Decision-making and Teacher's Support in Taiwan. J Comp Nurs Res Care 6(1):171. doi: https://doi.org/10.33790/jcnrc1100171.

Copyright: (C2020, This is an open-access article distributed under the terms of the Creative Commons Attribution License 4.0, which permits unrestricted use, distribution, and reproduction in any medium, provided the original author and source are credited.

\begin{abstract}
Background: The Taiwan Nursing Accreditation Council has proposed eight-core professional nursing qualities. Nursing students often encounter frustrations in clinical work, then they had more moral distress and reduce their moral sensitivity. They with moral sensitivity are aware of the correct behaviour, but may not be able to handle it properly when things come to a head. Then, student nurses, reduced their job satisfaction and transfer to other disciplines, or withdraw from school.
\end{abstract}

Objectives: To explore whether we can promote nursing students' moral sensitivity and reduce their moral distress in clinical environments by using the ethical decision-making model ( FourTopic Approach ) and teacher's support.

Research design: The study was an experimental research design. The participants were a randomized "experimental group" and a "control group". A pre-test and post-test involving the moral sensitivity and moral distress of subjects was administered before week 1 and after week 4. Experimental group intervention was performed by practicing the ethical decision-making model and giving teacher's support for 3 weeks. The comparison was made between the "experimental group" and the "control group" using moral sensitivity, moral distress. The 193 participants from juniorcollege student nurses. All participants were 18-20 years old. This study was accepted after institutional review boards of Kaohsiung Veterans General Hospital, nursing students were informed consent to participate in the proposed intervention.

Results: Comparison of the pre-test and post-test results revealed that the participants' moral sensitivity and moral distress scores increased significantly ( $p$ ?.005; p?.000). Comparison of the "experimental group" and "control group" in moral sensitivity and moral distress showed no difference ( $\mathrm{p}$ ?.05).

Conclusion: Nursing students who practiced ethical decisionmaking model ( Four -Topic Approach ) and got teacher's support, so nursing students need nursing ethics education in the internship stage is necessary.

\section{Introduction}

Nursing staff requires moral sensitivity (MS) to demonstrate professional competency because nurses with MSare self-aware of moral, ethical problem and their professional responsibilities [1]. Education institutes should aspire to foster student nurses' MS in hopes that they are aware of moral problem in clinical training [2], there by maximizing patients' well-being. Therefore, student nurses with higher MS naturally increased nursing care quality. How can student nurses'MS be raised? Student nurses can practice making ethical decisions to improve MS because ethical decision-making enables students to maintain correct thinking, which is crucial to engage in the correct behaviour. Cerit and Dinc [3] discovered that nurses that underwent bioethical decision-making practice were better at making moral judgments than those without undergoing such practice. Therefore, practice making ethical decisions facilitates the development of student nurses and enhances their moral sensitivity, there by maximizing patient benefits [4].

Nurses with MS are aware of the correct behaviour. However, such behaviour may be impeded by the lack of resources/support or by authoritarian oppression, causing moral distress (MD). Nursing staff that experience MD feel frustrated and guilty [5], demonstrate reduced job satisfaction, and question their role as a nurse [6]. In contrast, student nurses with MS are also aware of the correct behaviour, but may not be able to handle it properly when things come to a head. Similarly, student nurses, like nurses, may have their job satisfaction reduced and eventually lead to resignation [7]. In this context, student nurses may choose to suspend their study, transfer to other disciplines, or withdraw from school.

Yeh et al. [8] invited 44 Taiwanese students studying two-year nursing program to participate in focus interviews. The researchers found that students feel frustrated because of the inability help specific patients,students nurses' low professional status, being blamed and held responsible for mistakes made by doctors, head nurses, or nurses. Student nurses with unresolved MD will probably choose a different profession once they graduate. Therefore, we aspire to foster student nurses' independent thinking and group brainstorming, and enhance their self-learning abilities through ethical decisionmaking practice.If nursing students have more MD, whether they give up nursing education to transfer to non-nursing department, such as O'Connell [9] said nurses have more MD, they decreased job satisfaction and increased turnover. These abilities enable student nurses to confidently confront MD in clinical practice. Subsequently, education institutes can effectively reduce student nurses' MD by developing support networks that offer emotional support to student nurses, listens to their MD, and discusses questionable moral problem [10]. However, previous foreign and domestic studies largely adopted cross-sectional descriptive approaches to examine student nurses' MS and MD, and rarely are there studies that engaged in 
experimental research [2]. In this study, the Nursing Ethics Education Intervention Program (NEEIP) was developed and provided to student nurses to elucidate whether the program improved their MS and reduced their MD. In future, the program would expectantly improve student nurses' nursing competency and healthcare quality, thereby contributing to nursing ethics education.

Nurses are extremely shortage in Taiwan, Europe, and the United States [11]. The shortage of nurses, we should not only focus on retaining current nurses, but also encourage graduating student nurses to enter the profession. Each year, the placement of nursing graduates into relevant nursing-related positions is only $10.9 \%$ in Taiwan [12] suggesting that only a minority of nursing graduates truly assume nursing roles. The internship stage of nursing education is a vital time in which to enhance student nurses' willingness to enter the nursing industry, and the most effective way to learn about the workplace. Thus, it is essential that nursing education institutes formulate assistive plans for student nurses during their internship to reduce their MD. MD occurs when student nurses with favourable MS are unable to take correct actions. Failure to act correctly leads student nurses into an ethical dilemma, in which they are forced by circumstance or authoritarian oppression to engage in behaviours against their will [13]. Such experiential conflict and lack of support reduce student nurses' willingness to learn in the workplace, which may eventually lead them to forfeit their nursing career, transfer to another discipline or school, or withdraw from nursing school. By practicing making ethical decisions, student nurses can learn the integrity of ethical issues, foster their ethical judgement ability, and prevent MD [14]. Increased perceived support in the workplace elevates nurses' willingness to remain in the workplace, develop a support system, discuss sensitive or questionable moral problem [15], and hence reduce their MD [10]. Ke and Yang [16] advocated the training of expert ethical analysts to promptly provide consultation during clinical practice. The researchers also suggested the organization of support groups to confirm and clarify student nurses' values. In the present study, nursing ethics teachers were assigned to internship locations to provide consultation and support to student nurses during their internship period, thereby ensuring that student nurses maintain their passion for nursing and improving their healthcare quality.

\section{Objectives}

The study aimed to elucidate the changes in student nurses' MMSQSN and MD after they participated in the "Nursing Ethics Education Intervention Program (NEEIP) - Learning to Apply Ethical Decision (the Four-Topic Chart).

The objectives were as follows:

1. To explore the current conditions of MMSQ-SN and MD among Taiwanese nursing students;

2. To explorewhether the NEEIP intervention changed the MMSQ$\mathrm{SN}$ and MD conditions of student nurses;

3. To analyse the differences in the MMSQ-SN and MD between the experimental group (with NEEIP intervention) and the control group.

\section{Methods}

\section{Study design and participants}

The study was experimental research design. The present study adopted a purposive sampling method to recruit fifth-year student nurses in five-year nursing program currently in their internship stage. Student nurses that were not currently in an internship program or students that have not attended nursing ethics credits in the third year of study were excluded from the research. The participants were randomly allocated to an experimental group (two classes have 100 participants) and a control group (two classes have 100 participants). The two groups separately participated in a pre-test and a post-test. A ballot of five numbered balls (1, 2, 3, 4, and 5) was employed for random sampling. Participants that drew Ball 1 and Ball 3 were assigned to the experimental group and those that drew Ball 2 and Ball 4 were assigned to the control group. The experimental and control groups each comprised two classes with 100 participants. All participants attended a 4-hour course, specifically, "Nursing Ethics Education Intervention Program - Learning to Apply Ethical Decision (the Four-Topic Chart)," and were given written information. During the internship period, the nursing ethics teachers visited the experimental group participants at their internship location, and listened to their pour-out, made one face-to-face interview and one telephone interview of roughly 15 to 20 minutes each; each participant was visited by the nursing ethics teacher and their class teacher once. Participants in the control group only received one visitation by their class teacher during the internship period.

\section{Program design}

The NEEIP refers to the provision of the "Learning to Apply Ethical Decision (the Four-Topic Chart) intervention and nursing ethic teacher's support." The "Learning to Apply Ethical Decision (the Four-Topic Chart)" intervention entails the application of theoretical principles, specifically, the principles of beneficence and nonmaleficence concerning medical/nursing indications, the principle of respect and autonomy concerning patient preferences, the principles beneficence, nonmaleficence, and autonomy concerning quality of life, and the principles of good faith and justice concerning contextual features, to analyse ethical issues, which the student nurses employed to practice making ethical decisions. The nursing ethic teacher's support entails the provision of MMSQ-SN and emotional support to nursing interns by nursing ethics teachers via visits, listening to their concerns, and telephone interviews to show care.

\section{Instruments}

A literature review was conducted in the present study to collect the necessary data to develop a questionnaire draft. The content of the questionnaire was presented to a panel of experts to test its validity and reliability. The results were then used to develop a moral sensitivity questionnaire and moral distress questionnaire suitable for Taiwanese student nurses.

The original scale for the moral sensitivity questionnaire was developed by Swedish scholar, Lützén. Comire [2] obtained the permission of Lützén to modify his moral sensitivity to moral sensitivity questionnaire for student nurses (MMMSQ-SN). The modified questionnaire comprised six sections, namely expressing benevolence (EB), structuring moral meaning $(\mathrm{MM})$, interpersonal orientation (IO), experiencing conflict (EC), modifying autonomy (MA), and professional knowledge (PK). The MMMSQ-SN comprised 30 items. The items were scored on a Likert sevenpoint scale, where a score of 1 represents "strongly disagree" and 7 represents "strongly agree". The permission of Comire and Lützén was obtained to use the MMMSQ-SN in the present study. Comire suggested that the MMMSQ-SN be appropriately modified to fit Taiwanese culture. MMMSQ-SN was revised for Taiwan's nursing students, it 'Cronbach'salphais 0.899.

The permission of Eizenberg et al. [17] was obtained to use the Moral distress questionnaire (MDQ). The questionnaire comprised 15 items and it is suitable for a variety of job environments and cultures. MD is categorized into three scopes, specifically, (1) employees' job-related relationships, (2) lack of resources, and (3) time pressure. The scores of the 15 items are summed to represent MD levels. The items were scored on a Likert seven-point scale, where a score of 1 represents "not correlated" and 7 represents "highly correlated." The questionnaire achieved a validity score (Cronbach's alpha) of roughly 0.7 to 0.8 [17]. Items 5 and 10 of the original questionnaire entail the authoritarian oppression of doctors to engage in medical or nursing tasks. However, student nurses are typically instructed by anurse or clinical instructor in Taiwan. Thus, "doctor's instruction" was revised to "nurse's instruction" in Item 5. Item 15 entails the transferring 
of patients to unsuitable wards. This item is beyond the duty of student nurses. Thus, this item was revised to "providing inappropriate nursing care.'The revision Moral distress questionnaire in this study achieved a reliability level (Cronbach's alpha) of 0.814 .

\section{Ethical consideration}

The study protocol was approved by the institutional review boards of Kaohsiung Veterans General Hospital. The study contacted the administrative unit of nursing department to obtain permission to explain the research objectives and recruit students to participate in the proposed intervention. Participants under the age of twenty were required to submit their written consent and a written consent from a family member or legal guardian prior to participating in the intervention. Participants aged 20 or older were required to submit a written consent prior to participating in the intervention. Participants reserved the right to withdraw from the research at any time and their withdrawal did not affect their internship performance.

\section{Results}

\section{Participant demographic data}

A total of 193 participants were recruited, comprising 186 women (96.4\%) and 7 men (3.6\%). Among the participants, the majority were first in their order of birth $(n=75 ; 38.9 \%)$, were atheist $(n=93$; $48.4 \%$ ), lived with their parents ( $\mathrm{n}=105 ; 55.6 \%)$, lived in urban areas $(n=124 ; 64.9 \%)$, and were influenced by their mothers in term of professional ethics $(\mathrm{n}=131 ; 67.1 \%)$. In addition, most participants' primary influencers of professional ethics were college graduates $(\mathrm{n}=96 ; 50.5 \%)$ and worked as professionals $(\mathrm{n}=57 ; 30 \%)$. Moreover, most participants' greatest mental supporter were friends $(n=81$; $42 \%)$ and their mothers $(\mathrm{n}=85 ; 44 \%)$. Participants rarely participated in internships that discussed medical ethics (Table 1).

\begin{tabular}{|c|c|c|}
\hline item & $\mathbf{N}$ & $\%$ \\
\hline \multicolumn{3}{|l|}{ Gender } \\
\hline Male & 7 & 3.6 \\
\hline Female & 186 & 96.4 \\
\hline \multicolumn{3}{|l|}{ Birth Order } \\
\hline Only son / daughter & 21 & 10.9 \\
\hline First child & 75 & 38.9 \\
\hline Middle & 35 & 18.1 \\
\hline Last & 62 & 30.1 \\
\hline \multicolumn{3}{|l|}{ Religion } \\
\hline NO & 93 & 48.4 \\
\hline Buddhist & 41 & 21.4 \\
\hline Taoism & 46 & 24.0 \\
\hline Christian & 8 & 4.1 \\
\hline Yiguandao & 4 & 2.1 \\
\hline \multicolumn{3}{|l|}{ Lived with } \\
\hline parents & 105 & 55.6 \\
\hline father & 6 & 3.2 \\
\hline mother & 21 & 11.1 \\
\hline grand parents & 5 & 2.6 \\
\hline grand parents and parents & 26 & 13.8 \\
\hline hostel accommodation & 26 & 13.8 \\
\hline \multicolumn{3}{|l|}{ Living area } \\
\hline Downtown area & 124 & 64.9 \\
\hline Suburbs & 67 & 35.1 \\
\hline \multicolumn{3}{|c|}{ The education of key person impact values to NS ethical values } \\
\hline father & 73 & 37.8 \\
\hline Grand father & 1 & 0.5 \\
\hline mother & 131 & 67.9 \\
\hline Grand mother & 7 & 3.6 \\
\hline classmate & 69 & 35.8 \\
\hline tutor & 12 & 6.2 \\
\hline clinical teacher & 13 & 6.7 \\
\hline Illiterate & 2 & 1.1 \\
\hline Primary school & 10 & 5.3 \\
\hline Junior school & 17 & 8.9 \\
\hline High schools & 63 & 33.2 \\
\hline College & 96 & 50.5 \\
\hline Graduate School & 2 & 1.1 \\
\hline
\end{tabular}

Table 1. to be cont... 


\begin{tabular}{|l|l|l|}
\hline \multicolumn{2}{|l|}{ The occupation of key person impact to NS ethical values } \\
\hline unskilled workers & 23 & 12.1 \\
\hline Technical workers & 35 & 18.4 \\
\hline Semi-professional & 37 & 19.5 \\
\hline Specialty & 57 & 30.0 \\
\hline Senior Professional & 38 & 20.0 \\
\hline Mental support & \multicolumn{2}{l|}{} \\
\hline father & 67 & 34.7 \\
\hline Grandrand father & 1 & 0.5 \\
\hline classmate & 85 & 44.0 \\
\hline tutor & 13 & 6.7 \\
\hline clinical teacher & 16 & 8.3 \\
\hline The time of discussed medical ethics & \\
\hline Always & 9 & 5.1 \\
\hline often & 39 & 22.0 \\
\hline sometime & 57 & 32.0 \\
\hline seldom & 65 & 36.7 \\
\hline never & 7 & 40 \\
\hline
\end{tabular}

Table 1.Participant s'dataemographic data $(n=193)$

\section{Moral Sensitivity}

The majority of participant demographics (order of birth, religion, living with family, residence location, profession of influencer of professional ethics, frequency of discussion on medical ethics during internship, and internship field) were no significant differences with MMSQ-SN in both the pretest and the post-test ( $p>.05$ ). Only gender achieved significant differences with the MMSQ-SN in both the pre-test and the post-test $(\mathrm{p}=.04 ; \mathrm{p}=.03)$, where male exhibited a significantly higher MMSQ-SN than female. The education level of participants' influencer of professional ethics failed to achieve significant differences with MMSQ-SN in the pre-test but achieved significant differences with the MMSQ-SN in the post-test $(p=.02)$, where influencers with graduate degrees imposed the greatest influence on student nurses in terms of MMSQ-SN (Table 2).

\begin{tabular}{|c|c|c|c|c|c|c|}
\hline Item & Pre-test $\mathrm{M} \pm \mathrm{SD}$ & $\mathrm{t} / \mathrm{F}$ & $\mathrm{p}$ & Post-test $\mathrm{M} \pm \mathrm{SD}$ & $\mathrm{t} / \mathrm{F}$ & $\mathrm{p}$ \\
\hline Gender & & $-1.97^{*}$ & .04 & & $-2.17^{*}$ & .03 \\
\hline Male & $157.00 \pm 22.18$ & & & $162.71 \pm 20.46$ & & \\
\hline Female & $142.75 \pm 18.58$ & & & $146.65 \pm 19.18$ & & \\
\hline Birth order & & .37 & .77 & & 1.20 & .30 \\
\hline Only child & $143.42 \pm 16.62$ & & & $152.23 \pm 17.74$ & & \\
\hline First childFirst & $144.96 \pm 17.40$ & & & $148.49 \pm 21.03$ & & \\
\hline Middle & $141.48 \pm 19.31$ & & & $142.82 \pm 16.30$ & & \\
\hline Last & $142.17 \pm 21.11$ & & & $146.50 \pm 19.38$ & & \\
\hline Religion & & .83 & .50 & & .99 & .41 \\
\hline No & $143.91 \pm 18.39$ & & & $148.36 \pm 20.13$ & & \\
\hline Buddhist & $145.87 \pm 15.17$ & & & $145.04 \pm 16.25$ & & \\
\hline Taoism & $141.08 \pm 22.36$ & & & $145.17 \pm 20.00$ & & \\
\hline Christian & $143.25 \pm 16.54$ & & & $158.25 \pm 25.28$ & & \\
\hline Yiguandao & $130.50 \pm 26.23$ & & & $144.50 \pm 12.58$ & & \\
\hline Lived with & & 1.80 & .11 & & 1.24 & .29 \\
\hline parents & $140.82 \pm 17.15$ & & & $145.58 \pm 19.69$ & & \\
\hline father & $159.33 \pm 16.47$ & & & $162.00 \pm 22.85$ & & \\
\hline mother & $154.66 \pm 26.80$ & & & $149.47 \pm 15.21$ & & \\
\hline grand parents & $156.60 \pm 13.93$ & & & $149.00 \pm 14.74$ & & \\
\hline grand parents and patrents & $142.88 \pm 14.32$ & & & $144.11 \pm 14.40$ & & \\
\hline hostel accommodation & $143.69 \pm 22.07$ & & & $151.11 \pm 25.23$ & & \\
\hline Living area & & -1.24 & .21 & & -.47 & .63 \\
\hline Downtown area & $142.21 \pm 18.62$ & & & $146.79 \pm 19.68$ & & \\
\hline Suburbs & $145.76 \pm 19.12$ & & & $148.17 \pm 19.12$ & & \\
\hline
\end{tabular}




\begin{tabular}{|c|c|c|c|c|c|c|}
\hline $\begin{array}{l}\text { The education of key person impact to NS ethical } \\
\text { values }\end{array}$ & & .97 & .43 & & $2.65^{*}$ & .02 \\
\hline Illiterate & $150.00 \pm 19.79$ & & & $143.50 \pm 27.57$ & & \\
\hline Primary school & $131.80 \pm 11.78$ & & & $131.40 \pm 11.42$ & & \\
\hline Junior school & $145.64 \pm 12.92$ & & & $152.64 \pm 23.06$ & & \\
\hline High schools & $144.63 \pm 21.39$ & & & $151.61 \pm 17.86$ & & \\
\hline College & $142.96 \pm 18.79$ & & & $144.98 \pm 19.36$ & & \\
\hline Graduate School & $135.00 \pm 7.07$ & & & $155.00 \pm 36.76$ & & \\
\hline $\begin{array}{l}\text { The occupation of key person impact to NS ethical } \\
\text { values }\end{array}$ & & 1.23 & .29 & & 1.74 & .14 \\
\hline unskilled workers & $140.91 \pm 12.59$ & & & $152.91 \pm 18.62$ & & \\
\hline Technical workers & $142.28 \pm 18.16$ & & & $149.54 \pm 21.71$ & & \\
\hline Semi-professional & $145.16 \pm 20.24$ & & & $150.27 \pm 18.96$ & & \\
\hline Professional & $146.47 \pm 21.02$ & & & $145.22 \pm 20.62$ & & \\
\hline Senior Professional & $138.42 \pm 18.06$ & & & $141.78 \pm 15.58$ & & \\
\hline \multicolumn{7}{|l|}{ The time of discussed medical ethics } \\
\hline Always & $141.11 \pm 15.39$ & .42 & .78 & $148.66 \pm 11.62$ & .22 & .92 \\
\hline Often & $141.02 \pm 21.87$ & & & $148.07 \pm 21.94$ & & \\
\hline Sometime & $143.05 \pm 18.23$ & & & $146.26 \pm 18.90$ & & \\
\hline Seldom & $145.33 \pm 16.01$ & & & $145.69 \pm 16.32$ & & \\
\hline Never & $139.28 \pm 36.90$ & & & $151.28 \pm 32.76$ & & \\
\hline Department of nursing & & 1.04 & .40 & & .44 & .84 \\
\hline Medical-Surgical & $141.11 \pm 20.15$ & & & $149.88 \pm 25.10$ & & \\
\hline Long-term care & $149.63 \pm 16.63$ & & & $145.40 \pm 14.67$ & & \\
\hline Obstetric & $141.88 \pm 13.17$ & & & $145.22 \pm 17.04$ & & \\
\hline Pediatric & $149.82 \pm 31.37$ & & & $149.76 \pm 21.47$ & & \\
\hline Psychiatric & $141.94 \pm 15.33$ & & & $142.57 \pm 13.54$ & & \\
\hline Community & $145.38 \pm 18.46$ & & & $148.61 \pm 16.13$ & & \\
\hline
\end{tabular}

Table 2. Comparison with participant s' domparison with on MMSQ-SN( Pre-test \& Post-test )

\section{Moral distress}

The majority of participant demographics (order of birth, religion, living with family, education level of influencer of professional ethics, frequency of discussion on medical ethics during internship, and internship field) failed to achieve significant differences with MD in both the pre-test and the post-test. Only gender achieved significant differences with the MD in the pre-test $(\mathrm{p}=.04)$, where men exhibited a significantly lower level of MD than female. However, gender failed to achieve significant differences with MD in the post-test. Residence location achieved significant differences with MD in the pre-test $(\mathrm{p}=.02)$, where participants living in urban areas exhibited higher MD. However, residence location failed to achieve significant differences with MD in the post-test. The profession

\begin{tabular}{|l|l|l|l|l|l|l|}
\hline Item & Pre-test $\mathrm{M} \pm \mathrm{SD}$ & $\mathrm{t} / \mathrm{F}$ & $\mathrm{p}$ & Post-test $\mathrm{M} \pm \mathrm{SD}$ & $\mathrm{t} / \mathrm{F}$ & $\mathrm{p}$ \\
\hline Gender & & 1.70 & .04 & & 1.21 & .22 \\
\hline Male & $17.85 \pm 22.10$ & & & $26.57 \pm 18.08$ & & \\
\hline Female & $28.21 \pm 15.37$ & & & $33.91 \pm 15.43$ & & \\
\hline Birth order & & 1.79 & .15 & & 1.43 & .23 \\
\hline Only child & $31.12 \pm 18.58$ & & & $38.92 \pm 17.76$ & & \\
\hline First child & $28.69 \pm 14.43$ & & & $34.83 \pm 15.68$ & & \\
\hline Middle & $30.80 \pm 14.46$ & & & $34.23 \pm 14.34$ & & \\
\hline Last & $23.78 \pm 16.65$ & & & $29.93 \pm 15.45$ & & \\
\hline Religion & & 1.59 & .17 & & .44 & .77 \\
\hline No & $4.65 \pm 18.73$ & & & $7.18 \pm 16.08$ & & \\
\hline Buddhist & $-.80 \pm 15.54$ & & & $3.64 \pm 17.26$ & & \\
\hline Taoism & $3.95 \pm 23.10$ & & & $7.60 \pm 18.81$ & & \\
\hline Christian & $15.37 \pm 29.78$ & & & $.50 \pm 27.49$ & & \\
\hline Yiguandao & $14.75 \pm 16.09$ & & & $10.66 \pm 13.57$ & & \\
\hline
\end{tabular}




\begin{tabular}{|c|c|c|c|c|c|c|}
\hline Lived with & & .62 & .68 & & .49 & .78 \\
\hline parents & $28.23 \pm 16.71$ & & & $34.75 \pm 17.19$ & & \\
\hline father & $28.00 \pm 19.05$ & & & $32.66 \pm 16.65$ & & \\
\hline mother & $27.88 \pm 17.03$ & & & $29.27 \pm 13.37$ & & \\
\hline grand parents & $18.60 \pm 8.53$ & & & $28.75 \pm 12.86$ & & \\
\hline grand parents and parents hostel & $31.05 \pm 14.41$ & & & $31.61 \pm 11.59$ & & \\
\hline accommodation & $25.18 \pm 13.59$ & & & $35.05 \pm 15.29$ & & \\
\hline Living area & & 2.30 & .02 & & .40 & .68 \\
\hline Downtown area & $29.97 \pm 16.00$ & & & $33.97 \pm 15.74$ & & \\
\hline Suburbs & $24.04 \pm 14.78$ & & & $32.84 \pm 15.48$ & & \\
\hline $\begin{array}{l}\text { The education of key person impact to NS ethical } \\
\text { values }\end{array}$ & & 1.30 & .27 & & .55 & .73 \\
\hline Illiterate & 20.00 & & & 15.00 & & \\
\hline Primary school & $17.12 \pm 15.14$ & & & $34.60 \pm 15.29$ & & \\
\hline Junior school & $27.90 \pm 15.06$ & & & $33.41 \pm 15.79$ & & \\
\hline High schools & $26.48 \pm 13.25$ & & & $31.39 \pm 15.38$ & & \\
\hline College & $29.51 \pm 17.15$ & & & $34.82 \pm 16.12$ & & \\
\hline master & $341616 \pm 14.02$ & & & $37.50 \pm 12.02$ & & \\
\hline $\begin{array}{l}\text { The occupation of key person impact to NS ethical } \\
\text { values }\end{array}$ & & 3.04 & .01 & & .69 & .59 \\
\hline unskilled & $20.88 \pm 9.89$ & & & $35.50 \pm 14.22$ & & \\
\hline Technical & $22.40 \pm 12.70$ & & & $30.76 \pm 19.40$ & & \\
\hline Semi-professional & $34.33 \pm 18.12$ & & & $37.57 \pm 16.17$ & & \\
\hline Professional & $29.08 \pm 15.91$ & & & $33.61 \pm 14.09$ & & \\
\hline Senior Professional & $28.34 \pm 16.56$ & & & $31.38 \pm 15.12$ & & \\
\hline The time of discussed medical ethics & & 1.35 & .25 & & 1.22 & .30 \\
\hline always & $7.77 \pm 16.07$ & & & $6.00 \pm 22.46$ & & \\
\hline often & $7.48 \pm 23.37$ & & & $1.62 \pm 17.08$ & & \\
\hline sometimes & $3.17 \pm 20.33$ & & & $8.21 \pm 16.02$ & & \\
\hline seldom & $5.32 \pm 16.40$ & & & $6.26 \pm 15.92$ & & \\
\hline never & $13.57 \pm 28.57$ & & & $15.00 \pm 26.61$ & & \\
\hline $\begin{array}{l}\text { The education of key person impact to NS ethical } \\
\text { values }\end{array}$ & & 1.18 & .50 & & .83 & .54 \\
\hline Medical-Surgical & $27.06 \pm 14.04$ & & & $32.39 \pm 15.98$ & & \\
\hline Long-term care & $25.28 \pm 13.59$ & & & $34.05 \pm 17.72$ & & \\
\hline Obstetric & $27.23 \pm 20.31$ & & & $38.28 \pm 13.44$ & & \\
\hline Pediatric & $25.23 \pm 16.93$ & & & $28.86 \pm 15.07$ & & \\
\hline Psychiatric & $21.63 \pm 12.60$ & & & $31.38 \pm 14.45$ & & \\
\hline Community & $33.58 \pm 17.75$ & & & $38.09 \pm 15.55$ & & \\
\hline
\end{tabular}

Table 3. Comparison with participant s' on Moral distress( Pre-test \& Post-test )

\begin{tabular}{|l|l|l|l|l|l|}
\hline & team & $\mathrm{n}$ & $\mathrm{M} \pm \mathrm{SD}$ & $\mathrm{t}$ & $\mathrm{P}$ \\
\hline \multirow{3}{*}{ MS-pre } & control & 131 & $143.03 \pm 17.82$ & -.25 & .79 \\
\cline { 2 - 5 } & experiment & 62 & $143.77 \pm 21.00$ & & \\
\hline \multirow{3}{*}{ MS-post } & control & 131 & $148.00 \pm 19.63$ & .79 & .42 \\
\cline { 2 - 5 } & experiment & 62 & $145.61 \pm 18.99$ & & \\
\hline \multirow{2}{*}{ MD-pre } & control & 92 & $29.86 \pm 16.58$ & 2.14 & .03 \\
\cline { 2 - 5 } & experiment & 61 & $24.52 \pm 14.04$ & & \\
\hline
\end{tabular}




\begin{tabular}{|l|l|l|l|l|l|}
\hline MD-post & control & 82 & $33.80 \pm 15.95$ & .25 & .80 \\
\cline { 2 - 4 } & experiment & 53 & $33.11 \pm 15.16$ & & \\
\hline \multirow{2}{*}{ MS-dif } & control & 131 & $4.96 \pm 19.15$ & 1.04 & .29 \\
\cline { 2 - 4 } & experiment & 62 & $1.83 \pm 19.99$ & & \\
\hline \multirow{2}{*}{ MD-dif } & control & 75 & $4.70 \pm 15.87$ & -1.13 & .25 \\
\cline { 2 - 4 } & experiment & 53 & $8.24 \pm 19.31$ & & \\
\hline
\end{tabular}

Table 4. Compare the differences in moral sensitivity and moral distress between the experimental group and the control group

of influencer of professional ethics achieved significant differences with MD in the pre-test ( $p=.01)$, where semi-professional influencers imposed the greatest influence on the participants. However, the profession of influencer of professional ethics failed to achieve significant differences with MD in the post-test (Table 2).

In accordance with the principle of justice in learning, all nursing studentsare entitled to the right of education. Thus, all nursing interns are eligible to participate in the NEEIP. Results indicated that participants' MMSQ-SN significantly increased in the post-test, confirming the effectiveness of the NEEIP in improving student nurses' MMSQ-SN. However, results also showed that participants' MMSQ-SN also significantly increased in the post-test. Comparison of the pre-test and post-test results revealed that the participants' moral sensitivity and moral distress scores increased significantly $(\mathrm{p}=.005 ; \mathrm{p}=.000)$.Comparison of the "experimental group" and "control group" in Moral sensitivity and moral distress showed no difference $(\mathrm{p}=.05)$ (Table3).

The category of MMSQ-SN in pretest, the first, second and third was Professional Knowledge (PK), Modifying Autonomy (MA) and Interpersonal Orientation (IO), and then all nursing students took NEEIP, The order category of MMSQ-SN in the post test changed toIO, PK, and Expressing Benevolence (EB). Experiencing Moral Conflict (EC) ranked last among all students and refers to the spontaneous process that individual nursing students didn't use to solve a moral conflict. The mean score of EB, MM and IO in posttest were higher than mean score of EB, MM and IO in pretest. (Table4).

The average age of the participants was 19.39 years old. The age of the participants was significantly and positively correlated to the MMSQ-SNin the post-test $(\mathrm{p}<.01)$, suggesting that participants' MMSQ-SN scores in the post-test increased with their age. Moreover, the age of the participants was significantly and positively correlated to the difference between the pre-test and post-test MD scores $(p<.05)$. The experimental group and control group showed no significant difference $(\mathrm{p}>.05)$ in the post-test MMSQ-SN score, post-test MD score, difference between the pre-test and post-test MMSQ-SN scores, and difference between the pre-test and post-test MD scores. However, the MMSQ-SN and MD of the participants significantly increased following the teaching intervention $(p<.01)$. The pre-test MMSQ-SN score achieve a significant and positive correlation with the post-test MMSQ-SN score $(\mathrm{p}<.01)$ and the pretest MD score achieve a significant and positive correlation with the post-test MD score $(\mathrm{p}<.01)$. However, the post-test MD score failed to achieve significant correlation with the post-test MMSQ-SN score ( $>$ >.05).

\section{Discussion}

In accordance with the principle of justice, all nursing interns are entitled to the right of education. Thus, all nursing interns are eligible to participate in the "NEEIP - Learning to Apply Ethical Decision (the Four-Topic Chart)" intervention. However, results indicated that the experimental group and control group showed no significant difference in the post-test MMSQ-SN and MD scores. The difference between the experimental group and the control group was an additional visit from a teacher to provide support. However, student nurses may gain support from their family and friends. Since data about additional support from these sources were not collected, an additional visit from a teacher cannot be proven an effective intervention for the experimental group.

The results revealed significantly gender difference in the mean MMSQ-SN score in both the pre-test and the post-test ( $p=.04 ; \mathrm{p}=.03$ ), and the MD in the pre-test ( $\mathrm{p}=.04)$, Males exhibited a significantly higher MMSQ-SN than female, men exhibited a significantly lower level of MD than female. This MMSQ-SN score in gender results is difference to You et al study, but MD score in gender is consistent with O'Connell [9] study. These differentmaybe from culture, Males take more responsivities than female in Taiwan's culture.

Findings showed that the factors causing significant increases in MD were time pressure and job-related relationships. However, MD and MMSQ-SN failed to achieve significant correlations. Corley [18,19] asserted that MD occurs when responsible nurses are unable to engage in the correct behaviour due to circumstance or authoritarian oppression. Therefore, the definition of MD in the present study was different from "moral pressure" proposed by Lützén et al. [20], and thus the concepts were incomparable. However, the precursor to MD is the presence of MMSQ-SN, which enables student nurses to differentiate between right and wrong. In the present study, no significant correlation was observed between MD and MMSQ-SN, suggesting that there may be other factors influencing student nurses' MD and MMSQ-SN. Future studies can consider investigating the predictive factors of MD and MMSQ-SN.

The NEEIP intervention developed in the present study significantly enhanced students' MMSQ-SN and MD. Significantly improved areas of MMSQ-SN comprised IO, EB, and MM. IO showed the greatest improvement between the pre-test and post-test scores. This was consistent with the findings of Comrie [2], which examined student nurses' MMSQ-SN and found that female nurses were more likely to establish a favourable professional relationship with their patients. EB showed the second greatest improvement between the pre-test and post-test scores, suggesting that student nurses were more able to provide patient-centred care, improving patients' EB and comfort, thereby achieving "good nursing." MM refers to respecting patient's autonomy and being honest with patients. Morally inclined nurses are able to establish a trusting relationship with their patients and engage in the correct actions at the correct time. These three areas exhibited significant improvement because these can be more easily exhibited in their actions during their clinical internship.

\section{Limitation}

There are several limitations to this study, First, the study got limit resource, such as time and manpower, so only one nursing ethics teacher to visit nursing students and one teacher called to nursing students. Second, every nursing student practiced in different clinical work setting, the study lacked about work setting, practice setting data.Third, the study didn'tassess nursing students'clinical ethical climate, poor clinical ethical climate effects nursing students'moral sensitivity and moral distress.

\section{Conclusions}

In this study, we found that nursing students taking intervention 'Nursing Ethics Education Intervention Program - Learning to Apply Ethical Decision (the Four-Topic Chart) significantly increased 
the moral sensitivity after intervention, so nursing students need nursing ethics educationin the internship stage is necessary, but also they got more moral distress, and moral sensitivity was no relationship with moral distress, because teacher' support intervention was interview one time and call nursing studentsone time, so between the experimental group and the control group on moral sensitivity and moral distress were no significant difference, Because the source of support can come from family and classmates. The future research for social support mustmultiple way to collect social support. The result revealed males had more moral sensitivity than females, and less moral distress is lower than females, it's is different review paper. Nursing teacher or instructor should follow the nursing students that had more moral distress, should have appropriate support and consult, in order to help their internship can successfully passed,in the futurethey can become a professional nurses.

\section{Reference}

1. Lützén, K., Dahlqvist, V., Eriksson, S., \& Norberg, A. (2006). Developing the concept of moral sensitivity in health care practice. Nursing Ethics, 13, 187-196.

2. Comrie, R.W. (2012). An analysis of undergraduate and graduate student nurses' moral sensitivity. Nursing Ethics, 19,116-127.

3. Cerit, B. \& Dinc, L. (2012). Ethical decision-making and professional behavior among nurses: A correlational study. Nursing Ethics, 2, 200-212.

4. Numminen, O., \& Leino-Kilpi, H. (2007). Nursing students' ethical decision-making: A review of the literature, Nurse Education Today, 27, 796-807

5. Strandberg, G., Norberg, A., \& Jansson, L. (2003). Meaning of dependency on care as narrated by10 Patients. Research Theory Nursing Practice, 17, 65-/84.

6. Burston, A., \& Tuckett, A. (2013). Moral distress in nursing: Contributing factors, outcomes and interventions. Nursing Ethics, 20, 312-324.

7. Pauly, B., Varcoe, C., Storch, J., \& Newton, L. (2009). Registered nurses' perceptions of moral distress and ethical climate. Nursing Ethics, 16,561-573.

8. Yeh, M.Y., Wu, S.M., Che, H. L. (2010). Cultural and hierarchical influences: ethical issues faced by Taiwanese nursing students. Medical Education, 44,475-484.
9. O'Connel, C. B. (2015). Gender and the experience of moral distress in critical care nurses. Nursing Ethics, 22, 32-42

10. Unruh, J. A. (2010). Moral Distress: A Living Nightmare, Journal Emergency Nurses, 36, 253-255.

11. Zinn, J., Guglielmi, C., Davis, P., \& Moses, C. (2012). Addressing the nursing shortage: the need for nurse residency programs. AORN Journal, 96, 652-657.

12. Taiwan union of nurses association, 2016. Manpower reports. http://www.nurse.org.tw/DataSearch/ManpowerAll. aspx(accessed 14 Jun 2017).

13. Lützén, K, Cronqvist, A, Magnusson, A, Andersson, L. (2003). Moral stress: synthesis of a concept. Nursing Ethics, 10, 312322.

14. Sporrong, S.K., Hoglund, A.T., Arnetz, B. (2006). Measuring moral distress in pharmacy and clinical practice. Nursing Ethics, 13, 416-427.

15. Chen, H.C., C. I., Wang, Y.H., \& Lin, L. C. (2008). Turnover factors revisited: a Longitudinal Study of Taiwan-based staff nurses. International Journal of Nursing Studies, 45, 277-285.

16. Ko, H.K., \& Yang, M.S. (2009). Moral Distress and Leadership in Nursing. The Journal of Nursing, 56, 72-77.

17. Eizenberg, M., Desivilya, H., \& Hirschfeld, M. (2009). Moral distress questionnaire for clinical nurses: Instrument development. Journal of Advanced Nursing, 65, 885-892.

18. Corley, M. C., Minick, P., Elswick, P.K., \& Jacobs, M. (2005). Nurse moral distress and ethical work Environment. Nursing Ethics, 12, 381-390.

19. Corley, M. C. (2002). Nurse moral distress: A proposed theory and researchagender. Nursing Ethics, 9, 636-650

20. Lützén,K., Blom, T., Ewalds-Kvist, B.,... (2010). Moral stress, moral climate and moral sensitivity among psychiatric professionals. Nursing Ethics, 17, 213-222. 